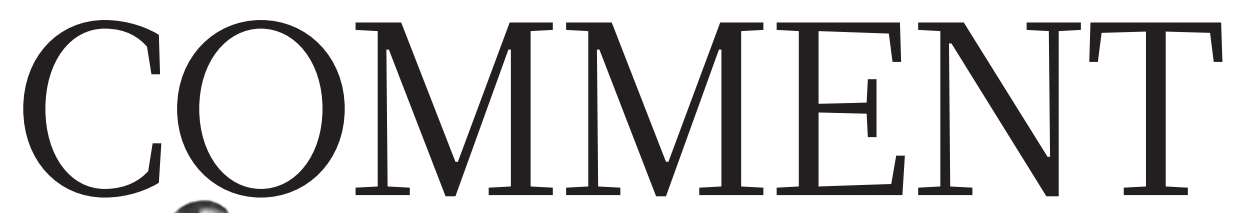

\begin{tabular}{l|l|l|l}
\hline $\begin{array}{l}\text { PoLICr Climate engineering } \\
\text { research and governance } \\
\text { needs to start small p.29 }\end{array}$ & $\begin{array}{l}\text { CORRESPONDENCE Lessons from } \\
\text { tefection of cold war } \\
\text { terrible toll of workaday } \\
\text { phicist revisited } \mathbf{p . 3 2}\end{array}$ & $\begin{array}{l}\text { OBITARY Mary F Lyon, } \\
\text { pioneer of mouse genetics, } \\
\text { remembered } \mathbf{p . 3 6}\end{array}$ \\
\hline
\end{tabular}

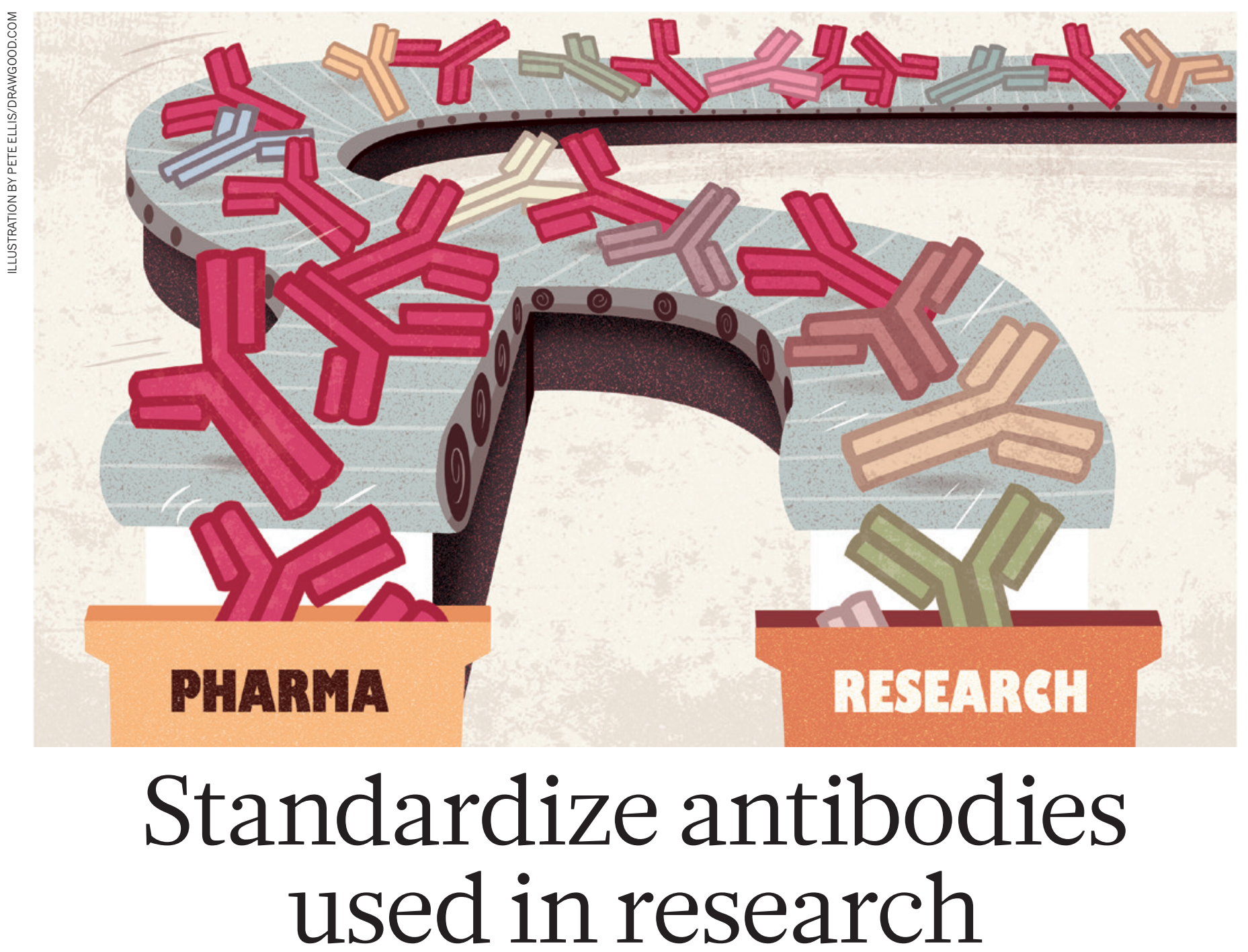

To save millions of dollars and dramatically improve reproducibility, protein-binding reagents must be defined by their sequences and produced as recombinant proteins, say Andrew Bradbury, Andreas Plückthun and 110 co-signatories.

\footnotetext{
$\mathrm{C}$

entral to reproducibility ${ }^{1}$ in biomedical research is being able to use reagents in publications. Alarmingly, there are serious flaws in the reliability of antibodies, the most widely used class of protein-binding reagent $^{2,3}$.

In the body, antibodies help to fight pathogens. In the lab, biologists have long used them to track proteins of interest because they
}

bind to specific targets. But in a 2008 study ${ }^{3}$, fewer than half of around 6,000 routinely used commercial antibodies recognized only their specified targets, with some manufacturers producing consistently good antibodies, and others consistently poor ones.

This figure may be optimistic ${ }^{4}$. In fact, we believe that poorly characterized and ill-defined antibodies were in large part to blame for a study co-authored by C. Glenn
Begley (a co-signatory to this article) being able to replicate the scientific results of only 6 of 53 landmark preclinical studies ${ }^{5}$. Across biomedical research, the resulting waste in materials, time and money is vast - costing an estimated US\$350 million annually in the United States alone.

To stem this loss, we call for an international collaboration and funding initiative to define all binding reagents 
$\checkmark$ according to the sequences that encode them. Crucially, researchers should use recombinant antibodies or binding reagents. These are made from reliable cell lines by isolating and incorporating the genes into plasmid DNA and transferring the plasmids into cells or bacteria for culture.

\section{WILDLY VARIABLE}

Researchers have used polyclonal antibodies for decades. These are produced by injecting a target (typically a protein) into an animal such as a rabbit and using the resulting serum as a source of antibodies. However, only $0.5-5 \%$ of the antibodies in a polyclonal reagent bind to their intended target. And functionality varies from batch to batch, because immunizing an animal - even the same one - never results in exactly the same mix of antibodies, making it hard for researchers to be sure of the specificity of any particular batch of binding reagent obtained in this way.

Four decades ago, the first monoclonal antibodies were made - by fusing a normal antibody-producing B-lymphocyte cell with a cancer cell to produce a 'hybridoma' ${ }^{6}$. The biomedical community believed that the resulting cell lines that produced (ideally) a single antibody species would solve many of the challenges of polyclonals. Unfortunately, monoclonals are far from problem-free.

Hybridoma cell lines can die off, lose their antibody genes, or simply not grow when taken out of frozen storage - meaning that the source of a particular monoclonal antibody may be lost forever. Furthermore, such antibodies may bind to more than one target, either because the antibody is actually a mixture of antibodies with multiple specificities, or simply because it is able to bind to several proteins. Careful characterization is thus required.

Most pharmaceutical and large biotechnology companies have whole departments dedicated to validating and characterizing antibodies. Consequently the reagents used in most clinical trials and especially in medical procedures cleared by the US Food and Drug Administration or the European Medicines Agency are extremely reliable.

Outside clinical trials, reagents are rarely validated to the same degree. What is more, only $44 \%$ of publications provide enough information - for instance, on the supplier for researchers to be able to purchase the same antibody ${ }^{7}$. The quality of the documentation that accompanies batches (such as on functionality in different assays) is enormously variable $^{2}$; even when it is provided, it may not correspond to the batch supplied ${ }^{8}$.

\section{TWO STEPS}

If all antibodies were defined by their sequences and made recombinantly, researchers worldwide would be able to use the same binding reagents under the same conditions. Immortal production lines of recombinant antibodies - which express no extra antibody chains - can be engineered by incorporating plasmids containing antibody DNA into cell lines.

In practice, improving the quality of protein-binding reagents will require two steps. First, the sequences should be obtained for widely used hybridoma-produced monoclonal antibodies. These antibodies should thence be produced recombinantly. (Polyclonal antibodies should be phased out of research entirely.)

Second, the research community should turn to methods that directly yield recombinant binding reagents that can be sequenced and expressed easily. These include display and two-hybrid methods - in which the best binders are selected from billions of variants - as well as approaches in which antibodies are identified from the sequencing of millions of animal or human B cells after immunological challenge.

Using sequence information as a universal reference system, researchers will be able to choose the binding reagent best suited to their requirements and use them in a standardized way (see 'Reliable binding reagents for all').
Various classes of binding reagents are being developed in addition to antibodies, including recombinant protein scaffolds - proteins with artificially introduced binding surfaces ${ }^{9}$ - and nucleic-acid-based binding reagents ${ }^{10}$. Some alternative reagents are easier to use and manufacture than antibodies.

\section{MARKET FORCES}

In our view, producing recombinant versions of the antibodies commonly used in research could be commercially profitable - even when the sequence information is publicly available. The absence of these reagents in the research-antibody marketplace stems mostly from economic considerations, rather than technical challenges. Most commercial producers have simply been attracted to the more lucrative therapeutics market.

Production costs for recombinant binding reagents - currently similar to those for monoclonals - should decrease as technologies improve, demand increases and processes become automated. Although researchers could, in principle, make the recombinant reagents themselves if the DNA sequences are freely available, most will prefer the convenience of receiving a qualitycontrolled product from a supplier. On this basis, two years ago, a UK start-up called Absolute Antibody began selling sequenced monoclonal antibody reagents produced recombinantly. Many of the sequences are already in the public domain.

That said, market forces alone are not going to improve the quality of protein-binding reagents - as the past 30 years have shown. Of the nearly 2 million antibodies listed in the database CiteAb, most are used too rarely in research to constitute an appealing commercial prospect.

Achieving a wholesale transition to characterized, recombinant protein-binding reagents will require the public funding agencies of the world's largest economies - North America, Europe and Asia - to make a major investment in technology development. This

RELIABLE BINDING REAGENTS FOR ALL Making the sequences of all binding reagents freely available would give researchers and suppliers a universal reference system.
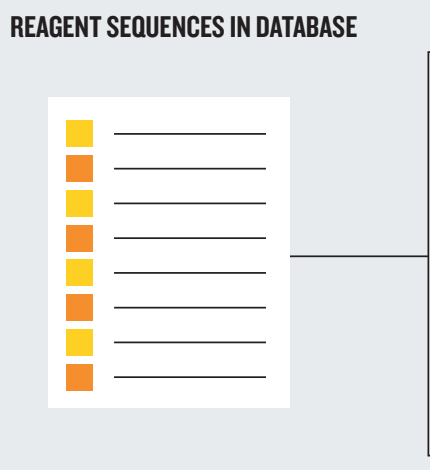

DISTRIBUTION METHODS

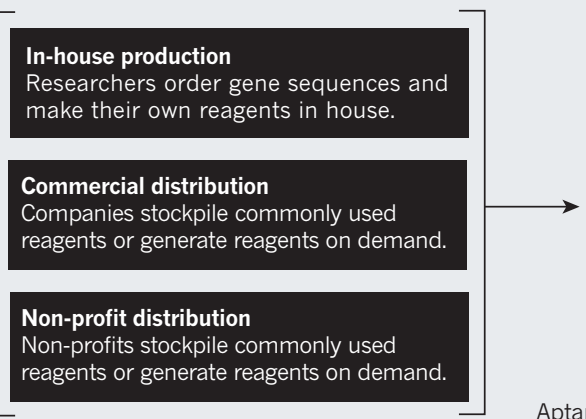

Aptamers (nucleic-acid-based reagent)

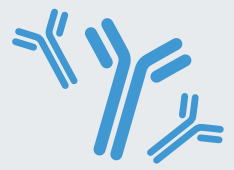

Recombinant monoclonal antibodies

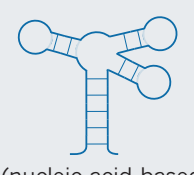

STANDARDIZED BINDING REAGENTS 
MONEY DOWN THE DRAIN The use of poorly characterized and ill-defined antibodies wastes materials, researcher time and money.

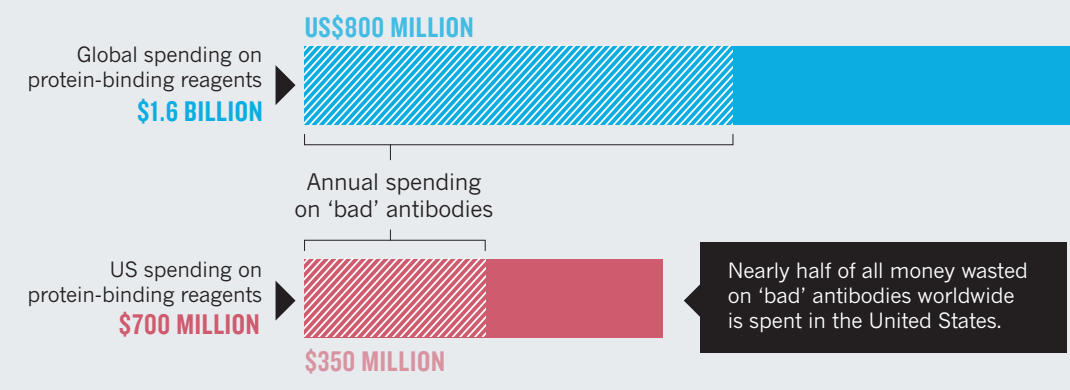

All costs estimates assume that $50 \%$ of antibodies are validated and that researchers buy 'bad' antibodies as often as they buy 'good' ones.

would reduce costs and increase efficiency in the key bottlenecks: the production of reagent targets, the selection of binding reagents and their downstream characterization. Five-year pilot programmes initiated in 2010 - the Protein Capture Reagents Program of the US National Institutes for Health (NIH) and Affinomics by the European Union (EU) have indicated that recombinant technologies could be scaled up. These programmes should be expanded and investment in them sustained for at least a decade.

We estimate that using current technology, roughly $\$ 1$ billion would be required to generate characterized recombinant binding reagents to target the primary products of all 20,000 human genes. This is probably less than what is wasted worldwide on bad reagents in two years (see 'Money down the drain'), and would be easily recouped over the long term thanks to more reproducible data.

However, we do not advocate the stockpiling of reagents against targets of minimal interest. Instead, efficient pipelines should be developed to generate any high-quality binding reagent on demand. One possibility is that centres funded by public and private funds would focus on target production, selection, characterization and the publishing of sequence information based on requests from users, whereas commercial companies would specialize in producing and distributing the reagents. An instructive analogue is the Structural Genomics Consortium. This partnership has over the past decade used public and private funds to generate effective production lines for human proteins, deployed by academia and pharmaceutical companies.

As a first step, we ask the scientific leadership of the NIH and the EU to convene academic users, technology developers, biotech companies, funding agencies and publishers, and establish a realistic timetable for the transition to these high-quality binding reagents.

Making sequenced well-characterized reagents is alone unlikely to change the behaviour of researchers. One possible outcome of such a meeting could be that publishers and funding agencies should mandate that in, say, five to ten years time, and contingent on appropriate investment, all binding reagents in published papers are recombinant and defined at the sequence level. This would mirror the requirements for the past few decades that gene sequences and coordinates for new protein structures be deposited and made publicly available.

If these steps are taken, scientists will not want to use unsequenced binding reagents, and the absence of sequencing information will lead to market disadvantages for vendors. The uncharacterized, unsequenced research antibody will become obsolete.

Andrew Bradbury is a group leader and scientist in the Bioscience Division, Los Alamos National Laboratory, Los Alamos, New Mexico, USA. Andreas Plückthun is professor and head of the Biochemistry Department of the University of Zurich, Switzerland.

e-mail:amb@lanl.gov

1. Collins, F. S. \& Tabak, L. A. Nature 505, 612-613 (2014).

2. Bordeaux, J. et al. BioTechniques 48, 197-209 (2010).

3. Berglund, L. et al. Mol. Cell Proteomics 7, 2019-2027 (2008).

4. Slaastad, H. et al. Proteomics 11, 4578-4582 (2011).

5. Begley, C. G. \& Ellis, L. M. Nature $483,531-533$ (2012).

6. Köhler, G. \& Milstein, C. Nature 256, 495-497 (1975).

7. Vasilevsky, N. A. et al. PeerJ 1, e148 (2013)

8. Voskuil, J. L. A. F1000Research 3, 232 (2014).

9. Binz, H. K., Amstutz, P. \& Pluckthun, A. Nat Biotechnol. 23, 1257-1268 (2005).

10.Gold, L. et al. Cold Spring Harb. Perspect. Biol. 4, a003582 (2012).

For a full list of co-signatories, see go.nature.com/ sws $5 f x$.

\section{Start research on climate engineering \\ Safe, small-scale experiments build trust and road-test governance, argue Jane C. S. Long, Frank Loy and M. Granger Morgan.}

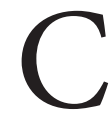
limate engineering - cooling Earth intentionally by modifying its radiation balance - worries many people. We know little about the effectiveness of these technologies or their side effects. The unintended consequences could be profound. One country's interventions will affect others and could distract from climate-change mitigation efforts, and there is no international mechanism for regulating such deployments. These are legitimate concerns.

But interventions may need to be considered in the future. The 2013 report of the Intergovernmental Panel on Climate Change suggested that even if the world almost eliminates greenhouse-gas emissions by mid-century, decades of climate engineering - such as removing carbon dioxide from the atmosphere or injecting reflective particles into the stratosphere might be required to control global temperatures and preserve vulnerable populations and ecosystems ${ }^{1}$.

Yet the climate-science community has largely avoided the subject. Governmentfunded research has been restricted to 